\title{
The Crossmodal Influence of Odor Hedonics on Facial Attractiveness: Behavioural and fMRI Measures
}

\author{
Francis McGlone, Robert A. Österbauer, \\ Luisa M. Demattè and Charles Spence \\ Additional information is available at the end of the chapter \\ http://dx.doi.org/10.5772/56504
}

\section{Introduction}

Facial attractiveness is a highly relevant social cue, readily assessed by human observers. Facial attractiveness significantly impact on success in both work and social environments [1, 2]. Taking a Darwinian perspective, Perrett at al. [3] have argued that the physical structure of beautiful faces - as judged by others - provide salient signals of mate value that motivate behavior in others. Several general features have been shown to contribute to the perceived attractiveness of a face, including both facial symmetry and the extent to which an individual face conforms to an average prototype $[4,5,6]$. Additionally, faces displaying various emotional expressions (e.g., joy, anger, etc.) have been used to investigate the brain regions involved in the coding of affect $[7,8,9,10,11]$, such as the orbitofrontal cortex (OFC), the insular cortex, and the amygdala.

At both an explicit and implicit level, humans through the ages have devised means by which to enhance facial attractiveness (a multibillion dollar cosmetic industry attests to this fact). An equally lucrative fragrance industry exploits the hedonic primacy of odors in the human brain, yet it remains unclear whether the presence of odors can modulate the perceived attractiveness of faces.

A pioneering positron emission tomography (PET) study by Nakamura and colleagues [12] demonstrated that activity in left frontal brain regions correlates with perceived facial attractiveness in humans. Furthermore, functional magnetic resonance imaging (fMRI) has been used to show that the viewing of attractive female faces by male participants activates reward circuitry in the brain, in particular, the nucleus accumbens and the OFC $[13,14]$. 
A recent study investigated the neural circuitry involved in the perception of facial attractiveness more directly by presenting participants with faces of varying attractiveness, while they performed a gender discrimination task [15]. Correlation analysis with ratings of attractiveness for the presented faces revealed a region in the medial orbitofrontal cortex (OFC) that responded specifically to facial attractiveness.

Physical attractiveness is not, however, solely dependent upon the visual aspects of appearance, but is often modulated by other sensory cues. For example, a person's voice has been shown to influence a speaker's perceived attractiveness [16, 17]. Similarly, a person's body odor also influences their perceived attractiveness [18]. The notion that odors can exert an influence over the perception of facial characteristics is also supported by the observation that the perceived masculinity/femininity of faces may be modulated by the presence of human sex hormone-like chemicals [19]. Additionally, the presence of a malodor can negatively influence the perceived attractiveness of male faces as rated by female observers in a psychophysical judgment task [20].

Only a few neuroimaging experiments have simultaneously presented odors and faces to participants, but none have directly assessed the impact of odor valence on facial attractiveness. For example, an fMRI study conducted by Gottfried and colleagues [21] paired faces with either a pleasant, unpleasant, or neutral odor, in an associative learning paradigm. Their results suggest that the brain regions involved in the processing of positive and negative affect, such as OFC, nucleus accumbens, and amygdala, are engaged during the appetitive and aversive learning process. Additionally, those brain areas previously found to participate in low-level odor processing, such as the piriform cortex and the caudal OFC, were also found to play an active role in the transfer of affective value between the olfactory and visual modalities. However, since no measure of the attractiveness of the faces was obtained, it remains unclear whether odor valence actually influenced the participants' perception of the faces.

Finally, it could be argued that odors do not necessarily modulate facial attractiveness per se, but rather other affective components of interpersonal perception such as, for example, perceived sympathy [22]. Alternatively, however, the presentation of the odor could also induce a general change in a person's mood (or emotional state) that might also be expected to alter facial attractiveness. Indeed, the psychological and physiological literature published to date supports the view that visual stimuli can influence olfactory perception while olfactory cues rarely influence visual perception $[23,24,25,26]$. It thus appears reasonable to assume that the simultaneous presentation of an odor will not change the visual characteristics of a face as such, but rather will primarily just changes people's affective reaction to it.

The main aim of the present study was therefore to investigate, both behaviorally and using fMRI, whether olfactory cues can modulate visual judgments of facial attractiveness. In particular, we investigated whether olfactory cues of differing hedonic value (i.e., pleasant vs. unpleasant) enhance and/or reduce the perceived attractiveness of male faces to female participants. Additionally, we selected an artificial body odor and a common male fragrance as the olfactory stimuli for their ecological relevance when paired with human faces. We hypothesized that the OFC, in particular, would show differential responses depending on the perceived attractiveness of the stimuli presented, since this brain region 
is activated by pleasant / unpleasant smells [27, 28, 29, 21, 30], and is also known to encode facial attractiveness [31, 15].

\section{Material and methods}

\subsection{Participants}

Twenty-one healthy right-handed female volunteers participated in this study (mean age 23 years, age range 19-29 years). All of the participants were non-smoking, had no history of nasal dysfunction or allergies to odors and each gave written informed consent after having received the written instructions concerning the study. Three participants had to be removed from the data analysis because of excessive head motion during the brain scanning session, and the data from two participants were discarded because they were unable to detect the presence versus absence of the odors at above chance levels ( $44 \%$ and $53 \%$ correct, respectively). Consequently, the data analysis at the group level included a total of 16 datasets. The study was approved by the Central Oxford Research Ethics Committee (C99.179).

\subsection{Stimuli and task}

Two odorants were used in this study, an artificial body odor (Thiol compound) and a popular male fragrance. The odors were diluted in $30 \mathrm{ml}$ of dipropylene glycol at concentrations of $0.0033 \%$ for the body odor and $0.5 \%$ for the male fragrance. The olfactory stimuli were delivered with a custom-built, computer-controlled olfactometer at a flow rate of 4 liters/ second, through Teflon tubes placed directly under the participant's nose. The participants were asked to breathe normally through their nose and to refrain from making any unduly strong sniffing movements. Clean medical air was delivered continuously through the olfactometer except during the delivery of the olfactory stimuli.

Twenty male faces taken from the standardized database developed by Perrett and his colleagues [3] were used as the visual stimuli. These faces have previously been rated for attractiveness on a 5-point rating scale. We used a subset of these faces, consisting of the 10 faces with the highest attractiveness ratings and the 10 faces with the lowest attractiveness ratings. Full screen color images of the faces were generated using a video projector located outside the scanner room and projected onto a translucent screen placed directly outside the bore of the magnet. A mirror fixed on the head coil allowed the participants to view the screen while lying in the scanner.

Each of the 20 faces was presented three times, once together with each of the two odors and once in the absence of any odor, resulting in the three conditions 'face-pleasant odor', 'faceunpleasant odor' and 'face-no odor'. Additionally, each odor was presented 10 times in the absence of any visual stimulus, resulting in a total of 80 trials being presented to each participant. The order of trials was randomized for each participant, with the sole constraint that the same face was never presented consecutively. 
At the beginning of each trial, the participants were visually cued by the presentation of a fixation cross to breathe in and detect the presence or absence of an odor which was delivered for $2500 \mathrm{~ms}$ following the onset of the visual cue (Figure 1). The faces were presented for $1000 \mathrm{~ms}$ starting $1500 \mathrm{~ms}$ after the onset of the odor stimuli. This lag between visual and olfactory stimuli was chosen on the basis of the results of a pilot study which had established that participants perceived the onset of both stimuli as concurrent when presented at this temporal delay.

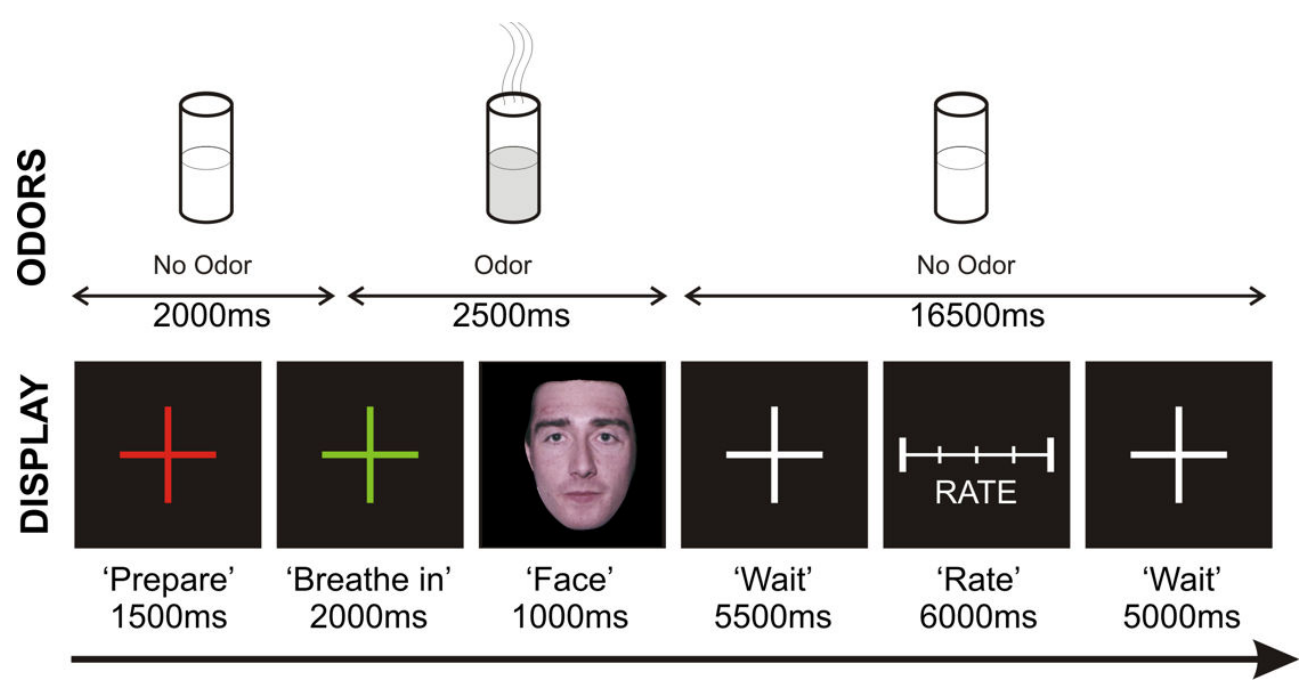

Figure 1. Participants were cued visually with a fixation cross that changed color from red to green to breathe-in and detect the presence or absence of an odor. In the trials where a face was presented, it was shown for $1000 \mathrm{~ms}$, beginning $2000 \mathrm{~ms}$ after the cue to breathe in. After a rest period of $5500 \mathrm{~ms}$, the participants had to rate the attractiveness of the face on a 5-point rating scale. When odors were presented in the same trial, odor stimulation started 500ms after the onset of the cue to breathe-in and lasted for $2500 \mathrm{~ms}$, terminating together with the face. When odors were presented without a face, the cue to breathe in was displayed for $3000 \mathrm{~ms}$ and the participants had simply to rate the pleasantness of the odor.

Following the presentation of the face, the participants rated its attractiveness on a rating scale ranging from $1=^{\prime}$ very unattractive' to $5=^{\prime}$ very attractive' with 3 as the neutral point. For the odor only trials, the participants had to rate the pleasantness of the odor on a similar scale ranging from $1=$ 'very unpleasant' to $5=$ 'very pleasant'. Behavioral measures relating to odor detection as well as the ratings were collected using a custom build button-box. The E-Prime software [32] was used to control stimulus presentation and to collect responses from the participants.

\subsection{Data acquisition and analysis}

Both functional and structural MRI images were acquired using a 3T Sonata Siemens scanner fitted with an 8-channel head coil (Siemens Medical Solutions, Erlangen, Germany) based at the University of Oxford Centre for Clinical Magnetic Resonance Research (OCMR). For the 
functional data series, a total of $1120 \mathrm{~T} 2 *$ weighted echo-planar imaging (EPI) volumes were taken over a time period of $28 \mathrm{~min}$.

Each volume consisted of 27 continuous oblique (tilted approximately $20^{\circ}$ upward from the anterior to posterior commissure line, so as to be aligned with the temporal lobes) slices of $3 \mathrm{~mm}$ thickness with an in-plane resolution of $3 \times 3 \mathrm{~mm}$. These imaging parameters allowed us to image the ventral two thirds of the brain until approximately Z-coordinate of +50 of the Montreal Neurological Institute (MNI) 152 standard brain space, to include all primary and secondary olfactory areas in the temporal lobes and OFC, as well as the visual cortex. Other imaging parameters were: TR=1.5s, 64x64 matrix, FOV 192×192 mm, TE=30ms and, flip angle $=90^{\circ}$.

After acquisition of the functional volumes, a $\mathrm{B}_{0}$ field map was acquired using a combined symmetric and asymmetric spin echo sequence. For registration into standard anatomical space, a single whole brain EPI volume (50 slices, TR=5s, other imaging parameters as above) as well as a high-resolution, whole-brain T1 weighted morphological scan (inversion-recovery fast gradient echo, $1 \mathrm{~mm}$ slice thickness, $1 \mathrm{~mm} \times 1 \mathrm{~mm}$ in-plane resolution) was acquired after the experimental paradigm had been completed.

Statistical image analysis of the functional dataset was carried out using the FMRIB Expert Analysis Tool (FEAT; www.fmrib.ox.ac.uk/fsl). The following pre-processing was applied: motion correction using MCFLIRT [33]; spatial smoothing using a Gaussian kernel of FWHM $5 \mathrm{~mm}$; mean-based intensity normalization of all volumes by the same factor; non-linear highpass temporal filtering (Gaussian-weighted LSF straight line fitting, sigma=25s). A general linear model using the conditions Face-No odor/Face-Body odor/Face-Fragrance/Body odor/ Fragrance as explanatory variables was fitted to the time course at each voxel. Statistical analysis for each experimental run was carried out using FMRIB's Improved Linear Model (FILM) with local autocorrelation correction [34].

For group analysis, the individual results were registered both to high-resolution anatomical MR images and to the Montreal Neurological Institute (MNI) 152 standard image. Registration to high resolution and standard images was carried out using FMRIB's Linear Image Registration Tool (FLIRT) [33]. Mixed-effects (often referred to as 'random-effects') group analysis was carried out using FMRIB's Local Analysis of Mixed Effects (FLAME) software [35] with a cluster threshold of $Z>2.0$ and a cluster significance threshold of $p=.05$ (corrected for multiple comparisons) [36, 37, 38].

\section{Results}

\subsection{Behavioral}

Comparison of the pleasantness ratings when the two odors were presented in isolation confirmed that the body odor was indeed perceived as significantly $[t(15)=8.04 ; p<.001]$ less pleasant $(M=1.78 ; S E M=0.14)$ than the fragrance $(M=3.68 ; S E M=0.22)$, as expected. The rating data for facial attractiveness were analyzed using a repeated-measures analysis of 
variance (ANOVA) with the factors of facial attractiveness (low vs. high) and odor condition (pleasant, unpleasant, or odorless). As expected, the results revealed a significant main effect of facial attractiveness, $[F(1,15)=138.23, p<.001]$, with participants judging the pre-selected attractive faces as being more attractive $(M=3.56$; $S E M=0.11)$ than those faces pre-selected to be less attractive $(M=2.15 ; S E M=0.1)$. Crucially, the main effect of odors on ratings of facial attractiveness was also significant $[F(2,14)=9.17, p<.01]$, demonstrating that the odors affected the perceived attractiveness of the male faces to the female participants. Post-hoc comparisons (Bonferroni corrected) of the 3 odor conditions revealed that participants rated the same faces as being significantly less attractive when presented together with the unpleasant body odor $(M=2.60 ; S E M=0.09)$ than when presented together with the pleasant odor $(M=2.99 ; S E M=$ $0.11 ; p<.01)$, or in the absence of any odor $(M=2.97$; $S E M=0.1 ; p<.01$; see Figure 2$)$. The analysis of this behavioral data revealed no significant difference in mean facial attractiveness ratings between the pleasant versus odorless conditions, nor any interaction between facial attractiveness and odor pleasantness $[F(2,14)<1 ; n . s$.$] .$

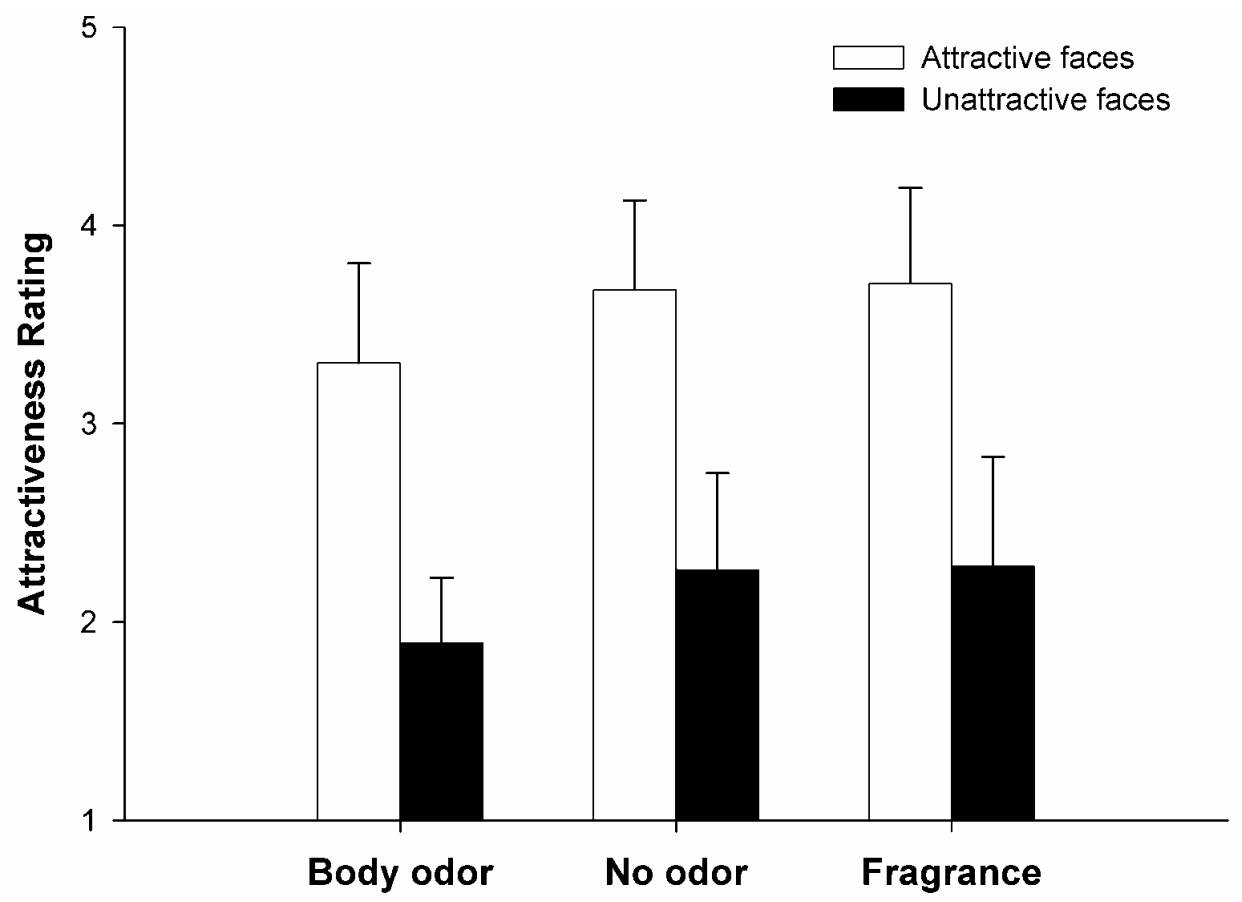

Figure 2. The average attractiveness ratings ( $n=16$ participants) for the same 10 faces a-priori assumed to be of high attractiveness compared to the 10 low attractiveness faces are shown when either presented with an unpleasant body odor, a pleasant fragrance, or in the absence of any specific odor. The difference in ratings between the attractive and unattractive faces was significant (paired t-test, $p<.05$ ) in each odor group. Additionally, faces presented together with the body odor were rated as significantly less attractive than those presented with the fragrance or those presented in the absence of any odor (paired t-test, $p<.05$ ) for both high and low attractive faces. 


\subsection{Neuroimaging data}

\subsubsection{Odor valence}

We first investigated those brain regions involved in encoding odor valence (pleasant vs. unpleasant) in the absence of any visual stimuli (i.e., faces). For this purpose, we computed two contrasts between the unimodal odor presentations, which were [Fragrance $>$ Body odor] and [Body odor $>$ Fragrance]. Results from the first contrasts showed several regions within the OFC to be more strongly activated by the pleasant fragrance as compared to the unpleasant odor (see Figure 3). These were located bilaterally in the medial OFC along the olfactory sulcus $(x / y / z=12 / 44 /-18 ; z$-score $=2.62$ and $x / y / z=-14 / 44 /-14 ; z$-score $=2.55)$, and in the lateral OFC in the right hemisphere only $(x / y / z=22 / 50 /-8 ; z$-score $=2.63)$. Additionally, a small cluster was detected in the right inferior frontal gyrus pars triangularis $(x / y / z=32 / 34 / 2 ; z$-score $=2.36)$.

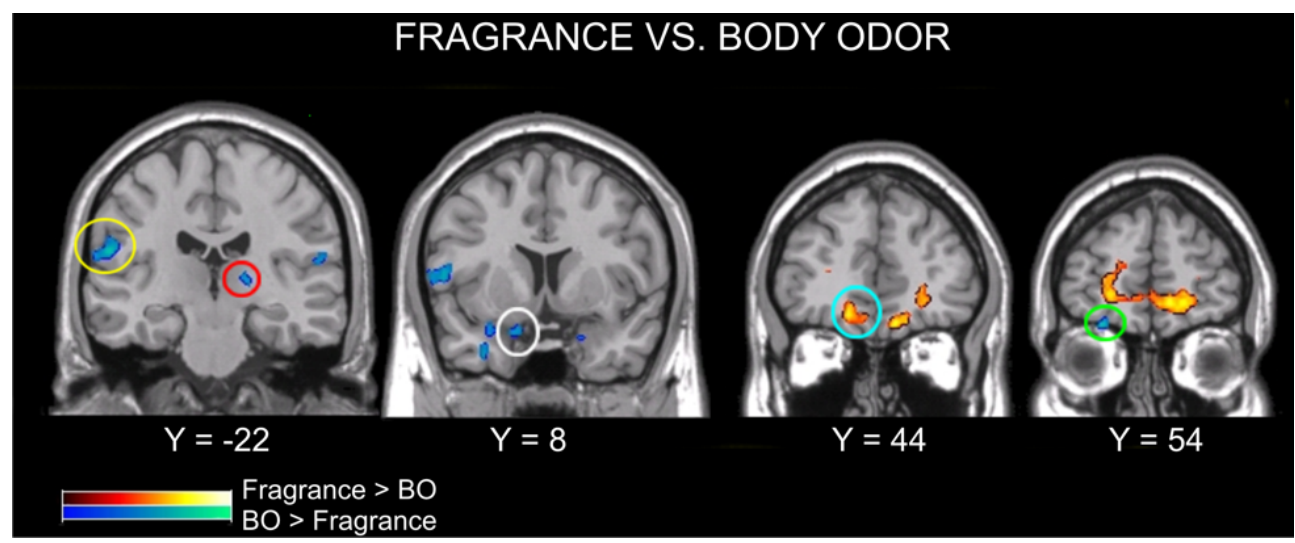

Figure 3. Group results ( $n=16$ participants) for brain regions showing differences in brain activation relating to the pleasantness of the odors are shown on coronal slices at different $y$-coordinates in the canonical MNI 152 space. The contrast [Fragrance $>\mathrm{BO}$ ] is rendered in orange/yellow and the contrast [ $\mathrm{BO}>$ Fragrance] is rendered in blue. The unpleasant BO elicited stronger responses in the supramarginal gyrus (yellow), thalamus (red), piriform cortex (white), and lateral OFC (green). In contrast, the pleasant fragrance caused stronger activation primarily in medial OFC (turquoise). The right side of each slice corresponds to the right side of the brain.

Conversely, within the $\mathrm{OFC}$, the unpleasant odor activated more strongly only in the left lateral orbital gyrus $(x / y / z=-24 / 54 /-18 ; z$-score $=2.51)$. However, activation was stronger in the primary sensory olfactory areas in the piriform cortex/amygdaloid area bilaterally $(x / y / z=20 / 10 /-26 ; z-$ score $=2.13$ and $x / y / z=-16 / 6 / 22 ; z$-score $=2.28)$ and in the frontal operculum $(x / y / z=58 / 0 / 10 ; z$ score $=2.13$ and $x / y / z=-54 / 8 / 8 ; z$-score $=2.33)$. Activation differences were also detected bilaterally in the supramarginal gyrus $(x / y / z=58 /-20 / 16 ; z$-score $=2.27$ and $x / y / z=-56 /-22 / 22 ; z$ score $=2.52)$ and in the right thalamus $(x / y / z=18 /-22 / 6$; $z$-score $=2.30)$.

\subsubsection{Facial attractiveness}

In order to highlight those brain areas involved in encoding the attractiveness of the face stimuli independently of odors, we compared the responses to faces with high vs. low 
attractiveness. For these contrasts, no distinction was made between the various odor conditions (Fragrance, Body odor and clean air), which were grouped together. The results revealed that irrespective of the odor condition, the presentation of the more attractive faces led to increased BOLD signal amplitude in the medial OFC in both hemispheres $(x / y / z=4 / 36 /-8 ; z$ score $=2.60$ and $x / y / z=-2 / 36 /-4 ; z$-score $=2.96$, see Figure 4$)$. Two other brain regions also showed stronger activation in response to the more attractive faces, namely the left nucleus accumbens $(x / y / z=-6 / 8 /-18 ; z$-score $=2.93)$ and the hypothalamus $(x / y / z=0 /-6 /-18 ; z$-score $=2.69)$. Conversely, the presentation of the unattractive faces resulted in stronger activation bilaterally in the amygdala $(x / y / z=22 / 0 /-12 ; z$-score $=2.19$ and $x / y / z=-22 /-4 /-14 ; z$-score $=2.45)$ as well as in the right pallidum $(x / y / z=26 /-10 /-6 ; z$-score $=2.99)$. Interestingly, the unattractive faces also elicited stronger activation in visual areas in the left inferior occipital gyrus $(x / y / z=-16 /-96 /-8$; $z$-score $=2.61$ ).

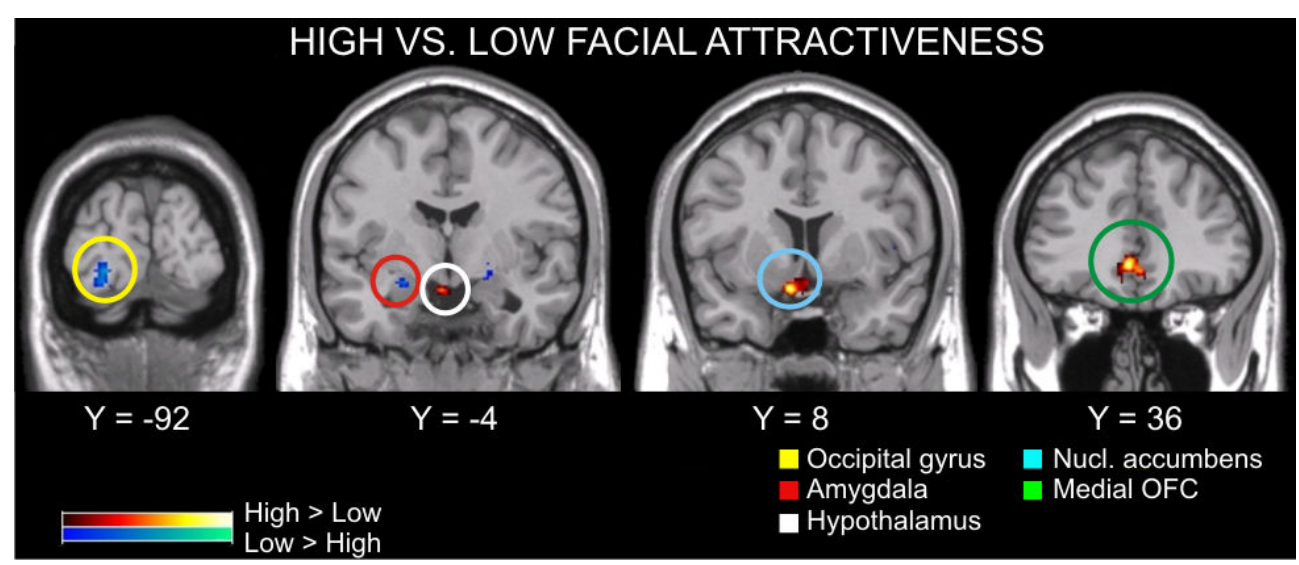

Figure 4. Group results ( $n=16$ participants) for brain regions showing differences in brain activation relating to the attractiveness of the faces (high vs. low) are shown on coronal slices at different $y$-coordinates in the canonical MNI 152 space. The contrast [High > Low] is rendered in orange/yellow and the contrast [Low > High] is rendered in blue. Attractive faces preferentially engaged the medial OFC, whereas the less attractive faces led to stronger activation in the amygdala. The right side of each slice corresponds to the right side of the brain.

\subsubsection{Odor-face interactions}

The primary interest of the present study was to address the question of whether or not odor hedonics would influence the perceived or implicit attractiveness of male faces to female participants. The behavioral results confirmed that the same set of faces was rated as being significantly more attractive when accompanied by the pleasant odor as compared to the unpleasant odor. We were specifically interested in the brain regions associated with this effect and therefore contrasted those trials in which the faces were presented with the pleasant male fragrance to those where the same faces were presented with the unpleasant body odor. Similar to the unisensory effects of pleasant vs. unpleasant odors and attractive vs. unattractive faces, the face stimuli caused significantly stronger activation in the medial $(x / y / z=-6 / 44 /-24 ; z$ - 
score $=2.64)$ and lateral $(x / y / z=-38 / 54 /-16 ; z$-score $=2.93)$ OFC as well as in the ventral striatum $(x / y / z=-2 / 12 /-16 ; z$-score $=2.63)$, when they were presented together with the pleasant odor (see Figure 5). A further analysis of the percentage BOLD signal change in the peak activated voxels in the left OFC (see Figure 6) revealed positive signal changes only when faces were presented with the fragrance or in the absence of odors. The responses in these regions to all other stimuli were either close to zero or slightly negative.

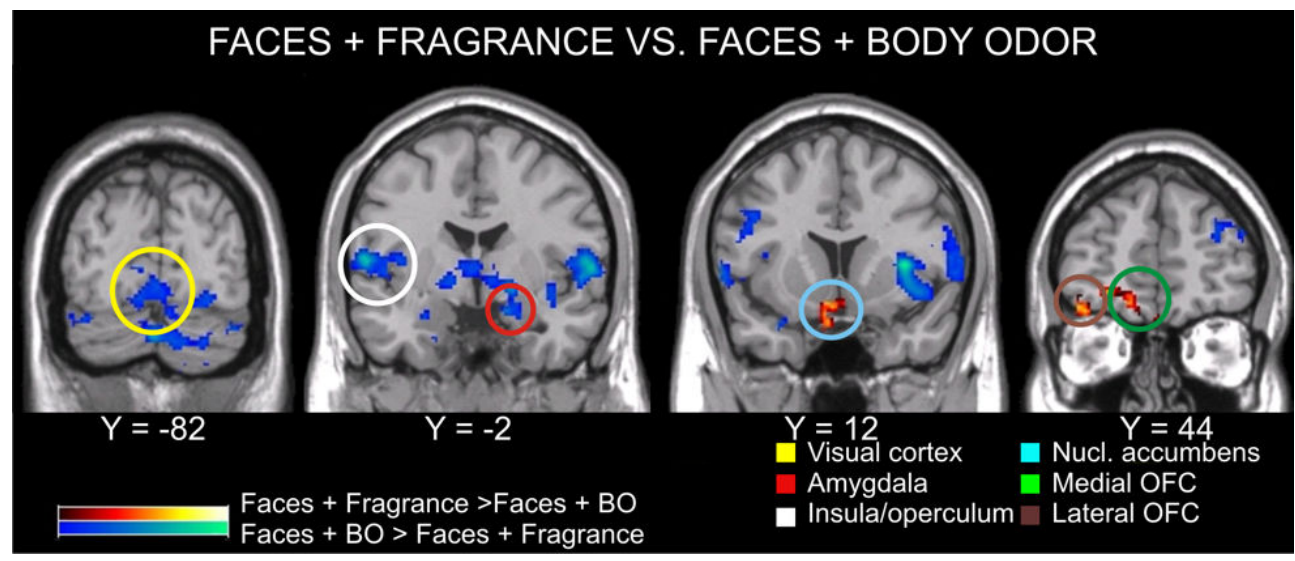

Figure 5. Group results ( $n=16$ participants) for brain regions showing differences in brain activation when the same set of faces was presented together with the pleasant fragrance compared to the unpleasant body odor. The contrast [Faces + Fragrance $>$ Faces $+\mathrm{BO}$ ] is rendered in orange/yellow and the contrast [Faces $+\mathrm{BO}>$ Faces + Fragrance] is rendered in blue. The presence of the fragrance preferentially engaged the OFC and ventral striatum. Conversely, the unpleasant odor caused stronger activation in the amygdala, insular cortex, and visual cortex. The right side of each slice corresponds to the right side of the brain.

Conversely, a different network of brain regions responded more strongly when the faces were presented together with the unpleasant body odor. The presence of the unpleasant odor caused significantly stronger activation in the amygdala $(x / y / z=20 /-8 /-16 ; z$-score $=3.03$ and $x / y / z=$ -18/-6/-16; $z$-score $=2.65)$ and anterior insular cortex $(x / y / z=36 / 12 / 6 ; z$-score $=3.95$ and $x / y / z=$ $-32 / 24 / 0 ; z$-score $=3.01)$. Furthermore, we observed significantly stronger responses in the thalamus $(x / y / z=12 /-16 / 4 ; z$-score $=3.95$ and $x / y / z=-6 /-20 / 4 ; z$-score $=3.39)$ and an extensive cluster located at the junction of the rolandic operculum with the superior temporal sulcus $(x /$ $y / z=60 /-22 / 18 ; z$-score $=4.06$ and $x / y / z=-62 /-20 /-14 ; z$-score $=3.79)$. The only differences in frontal brain regions was found in the right medial frontal gyrus $(x / y / z=30 / 28 / 30 ; z$-score $=2.69)$. Interestingly, both visual cortical areas $(x / y / z=-2 /-86 /-10 ; z$-score $=3.02)$ as well as the cerebel$\operatorname{lum}(x / y / z=12 /-56 /-14 ; z-s c o r e=3.50$ and $x / y / z=-20 /-60 /-20 ; z$-score $=3.97)$ also displayed stronger activation when the faces were accompanied by the unpleasant odor. A further analysis of the percentage BOLD signal change in the peak activated voxels in the amygdala (see Figure 7) revealed positive signal changes for all conditions. 


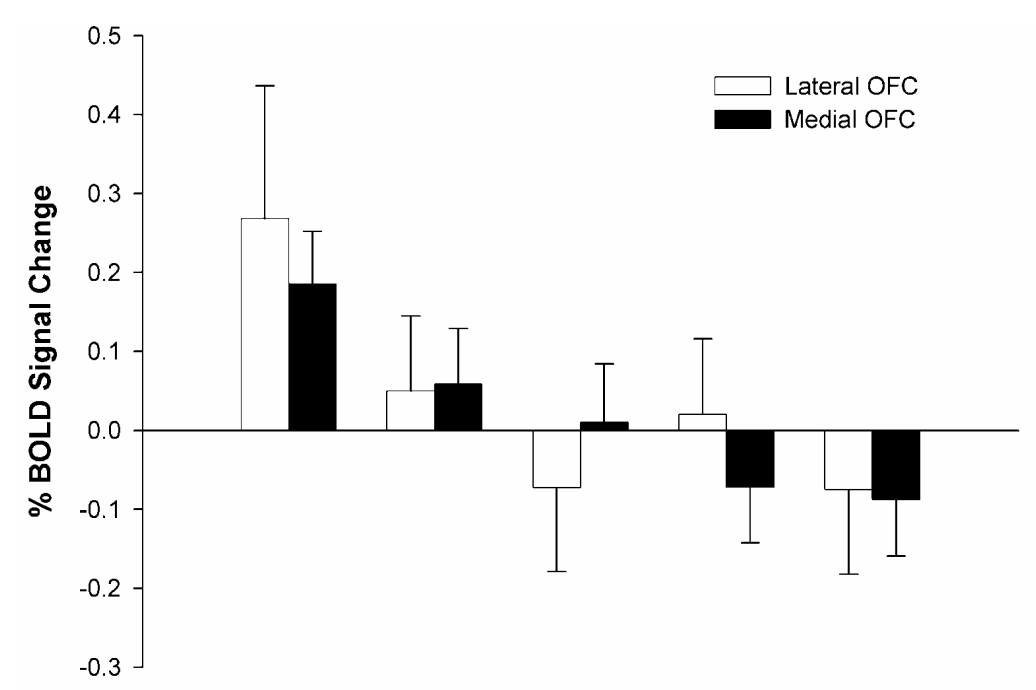

Face + Fragrance Face + clean air Face + Body odor Fragrance Body odor

Figure 6. The average ( $n=16$ participants) percentage BOLD signal change in the peak voxels in both medial and lateral OFC are shown for each of the five experimental conditions. Error bars depict the standard error.

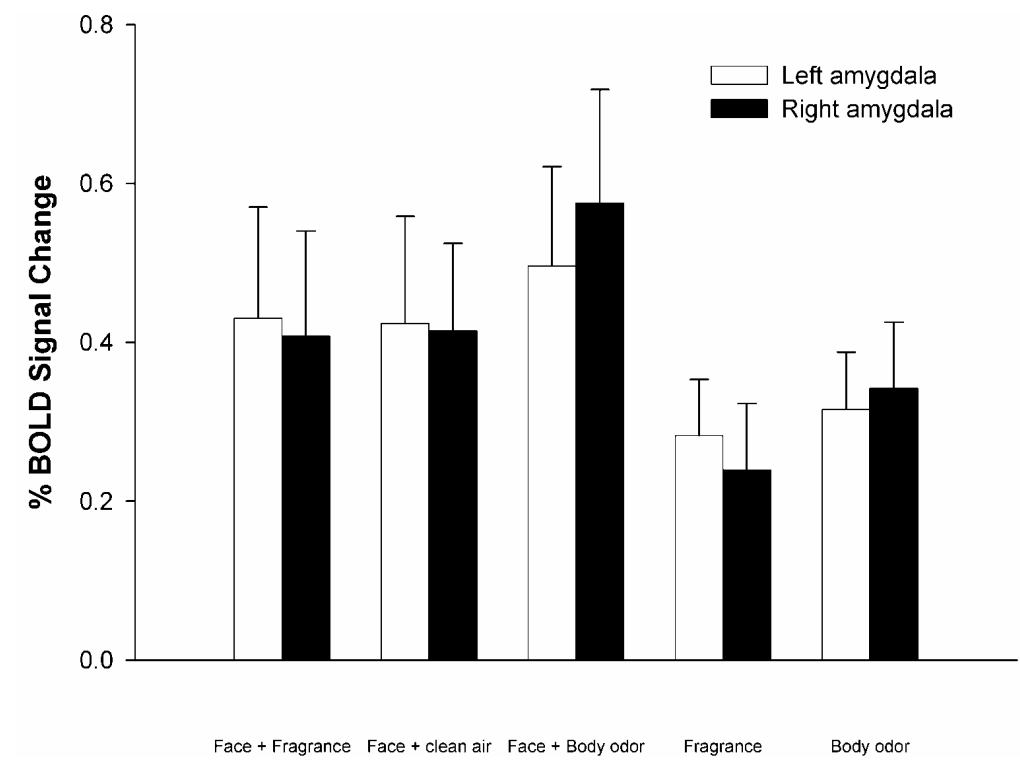

Figure 7. The average ( $n=16$ participants) percentage BOLD signal change from baseline in the peak voxels in both the left and right amygdala are shown for each of the five experimental conditions. Error bars depict the standard error. 


\section{Discussion}

The principal aim of the present study was to determine whether specific pleasant vs. unpleasant odors can exert a significant influence on the perception of facial attractiveness. Behavioral results confirmed that the two odors used in this study (a male fragrance and a synthetic body odor) were indeed perceived as different with respect to their pleasantness. Significantly, when briefly presented with the same set of male faces, the female participants in this study judged the faces accompanied by the pleasant fragrance as more attractive than when the faces were presented with an unpleasant synthetic body odor. This effect was related to a modulatory effect exerted by the unpleasant odor, as no significant difference in facial attractiveness ratings was found between the pleasant odor and a 'no odor' control condition.

In agreement with the behavioral data, the pleasant fragrance activated the medial OFC, a region that encodes the reward value of stimuli across a variety of sensory modalities including olfaction $[27,30,39,40]$. Conversely, the unpleasant odor activated a different network or brain regions, including the amygdala which has previously been implicated in the processing of aversive stimuli [30, 41, 42].

There is currently some controversy over the role of the amygdala in the processing of odor hedonics, since the established view that this region specifically encodes aversive odors [43, 42] has been challenged by several more recent studies. For example, Anderson and colleagues [27] used the pleasant odor citral (which has a lemon smell) and the unpleasant odor valeric acid (a rancid smell) in high and low concentrations so that they could vary the valence and intensity of these stimuli. They found that amygdala activation was associated with odor intensity, but was independent of valence. Conversely, activity in the OFC was associated with odor valence, independent of intensity. In a similar study using a greater number of odors (3 pleasant and 3 unpleasant), Rolls and colleagues [40] found that ratings of odor intensity were correlated with the magnitude of the BOLD signal in medial olfactory cortical areas (including the piriform and anterior entorhinal cortex), but not in the OFC. In contrast, pleasant odors were found to activate a medial region of the $\mathrm{OFC}$, whereas unpleasant odors activated the left lateral OFC, irrespective of odor intensity. Activation of this area have also been reported after monetary losses, unattractiveness in face stimuli, and the presentation of aversive odors $[39,44,45,46]$. Since the odors used in the present study were matched with respect to their intensities, this supports the notion that stimulus aversiveness is encoded in the human amygdala. This view is also supported by the results of a recent study demonstrating that the amygdala does not encode odor valence or intensity per se, but rather appears to contain a general representation of the emotional value of a stimulus [47].

When the activation seen in response to more attractive faces was compared to that seen in response to relatively less attractive faces, it was found that the former engaged a reward circuit consisting of the medial OFC and nucleus accumbens, consistent with previous studies [31, $14,15]$. By contrast, relatively less attractive faces gave rise to stronger activation in the amygdala, a region implicated in the processing of the emotional expression of faces $[48,49]$ particularly when the expression is negative (fear, anger). The results presented here suggest 
that less attractive faces produce a similar neural response to that elicited by faces displaying negative emotions.

The main finding to emerge from this study was that the presence of odors with different hedonic characteristics altered the perception of male facial attractiveness in female participants. Under such conditions of bimodal stimulation, we found that when the faces were presented together with a pleasant fragrance, increased BOLD activation was predominantly observed in the OFC and ventral striatum (i.e. in the same regions that are engaged when viewing attractive faces). It appears that the positive valence of the odor interacted with the representation (itself not unpleasant), with the neuroimaging data providing a more in these regions, leading to an overall positive emotional response to the multisensory combination of stimuli (i.e., face plus odor). This observation is consistent with previous studies that have implicated these regions in the processing of facial attractiveness [31, 14, 15] and positively valenced odors [27, 40,47]. Despite the observation that attractiveness ratings were no different when faces were presented together with the pleasant odor compared to faces presented in the absence of odors, significantly positive BOLD responses within the OFC were only observed in the former condition. This may suggest that even though the pleasant odor did not increase the consciously perceived and reported visual attractiveness of the faces per se, the medial OFC did and here we conjecture that this effect engaged more implicit affective processes that participants were unable to access consciously, but would nonetheless impact on mechanisms underpinning liking.

In contrast, when the male faces were presented with the unpleasant body odor, ratings of facial attractiveness were significantly reduced, compared to the pleasant odor and the odorless condition. The presence of the unpleasant odor caused significantly stronger activation in the insular cortex and the amygdala, both of which have previously been implicated in the representation of negative affect [50,51,52] and facial unattractiveness [15]. It thus appears that the aversiveness of the body odor negatively influences the emotional response to a face, leading to a decrease in its perceived attractiveness, an effect that was observed for faces of both high and low attractiveness. Several factors might explain the observation that the body odor influenced the ratings and brain activation more strongly than the fragrance. First, the hedonic difference of the body odor from hedonically neutral was greater than that of the fragrance (rating of 1.78 for body odor versus. 3.68 for fragrance with 3 being 'neutral'), so that a stronger effect might be expected on that basis alone.

In conclusion, even though the behavioral response (as measured by overt rating of facial attractiveness) in the presence of the pleasant odor, or no odor, condition did not influence facial attractiveness, results from the neuroimaging component of the study did show activations in the reward processing areas of $\mathrm{OFC}$ and ventral striatum. Reward region activity as evaluated using fMRI did not therefore follow the results of the behavioural task. The latter, we suggest, lacked the sensitivity required to dissociate between the fragrance and clean air (itself not unpleasant) of facial attractiveness with the neuroimaging data providing a more sensitive measure of affective state. For the unpleasant body odor, our findings support the notion that in the context of facial beauty, unpleasant odors have higher overt emotional salience than pleasant odors. 


\section{Acknowledgements}

We would like to thank Unilever Research for supporting this research.

\section{Author details}

Francis McGlone ${ }^{1}$, Robert A. Österbauer², Luisa M. Demattè ${ }^{3}$ and Charles Spence ${ }^{3}$

1 School of Natural Sciences and Psychology, Liverpool John Moores University, UK

2 Oxford Centre for Functional Magnetic Resonance Imaging of the Brain, John Radcliffe Hospital, University of Oxford, Oxford, UK

3 Crossmodal Research Laboratory, Department of Experimental Psychology, University of Oxford, Oxford, UK

\section{References}

[1] Frieze IH, Olson JE and, Good DC, Perceived and actual discrimination in the salaries of male and female managers. J. Appl. Soc. Psychol 20: 46-67, 1990

[2] Marlowe CM, Schneider SL, and Nelson CE. Gender and attractiveness biases in hiring decisions are more experienced managers less biased? J. Appl. Psychol 81: 11-21, 1996

[3] Perrett DI, Lee KJ, Penton-Voak I, Rowland D, Yoshikawa S, Burt DM, Henzi SP, Castles DL, and Akamatsu S. Effects of sexual dimorphism on facial attractiveness. Nature 394: 884-887, 1998.

[4] Grammer K, and Thornhill R. Human (Homo sapiens) facial attractiveness and sexual selection: the role of symmetry and averageness. J Comp Psychol 108: 233-242, 1994.

[5] Hoss RA, Ramsey JL, Griffin AM, and Langlois JH. The role of facial attractiveness and facial masculinity/femininity in sex classification of faces. Perception 34: 1459-1474, 2005.

[6] Perrett D, May K, and Yoshikawa S. Facial shape and judgments of female attractiveness. Nature 368: 239-242, 1994.

[7] Adolphs R, Tranel D, Damasio H, and Damasio A. Impaired recognition of emotion in facial expressions following bilateral damage to the human amygdala. Nature 372: 669-672, 1994. 
[8] Blair RJ, Morris JS, Frith CD, Perrett DI, and Dolan RJ. Dissociable neural responses to facial expressions of sadness and anger. Brain 122: 883-893, 1999.

[9] Haxby JV, Hoffman EA, and Gobbini MI. Human neural systems for face recognition and social communication. Biol Psychiatry 51: 59-67, 2002.

[10] Morris JS, Frith CD, Perrett DI, Rowland D, Young AW, Calder AJ, and Dolan RJ. A differential neural response in the human amygdala to fearful and happy facial expressions. Nature 383: 812-815, 1996.

[11] Posamentier MT, and Abdi H. Processing faces and facial expressions. Neuropsychol Rev 13: 113-143, 2003.

[12] Nakamura K, Kawashima R, Nagumo S, Ito K, Sugiura M, Kato T, Nakamura A, Hatano K, Kubota K, Fukuda H, and Kojima S. Neuroanatomical correlates of the assessment of facial attractiveness. Neuroreport 9: 753-757, 1998

[13] Aharon I, Etcoff N, Ariely D, Chabris CF, O'Connor E, and Breiter HC. Beautiful faces have variable reward value: fMRI and behavioral evidence. Neuron 32: 537-551, 2001.

[14] Kampe KK, Frith CD, Dolan RJ, and Frith U. Reward value of attractiveness and gaze. Nature 413: 589, 2001.

[15] O'Doherty J, Winston J, Critchley H, Perrett D, Burt DM, and Dolan RJ. Beauty in a smile: the role of medial orbitofrontal cortex in facial attractiveness. Neuropsychologia 41: 147-155, 2003.

[16] Casey SJ, Woods AT, and Newell FN. Is beauty in the eyes and ear of the beholder? In: 7th Annual Meeting of the International Multisensory Research Forum. Dublin, Ireland: 200

[17] Zuckerman M, Miyake K, and Hodgins HS. Cross-channel effects of vocal and physical attractiveness and their implications for interpersonal perception. J Pers Soc Psychol 60: 545-554, 1991.

[18] Rikowski A, and Grammer K. Human body odour, symmetry and attractiveness. Proc Biol Sci 266: 869-874, 1999.

[19] Kovács G, Gulyas B, Savic I, Perrett DI, Cornwell RE, Little AC, Jones BC, Burt DM, Gal V, and Vidnyanszky Z. Smelling human sex hormone-like compounds affects face gender judgment of men. Neuroreport 15: 1275-1277, 2004.

[20] Dematte ML, Osterbauer R, and Spence C. Olfactory cues modulate facial attractiveness. Chem Senses 32: 603-610, 2007.

[21] Gottfried JA, O'Doherty J, and Dolan RJ. Appetitive and aversive olfactory learning in humans studied using event-related functional magnetic resonance imaging. $J$ Neurosci 22: 10829-10837, 2002. 
[22] König R. Kulturanthropologische Betrachtungen zum Problem der Parfmierung [A cultural anthropological consideration of the problem of perfume]. J Cosmet Sci 23: 823-829, 1972.

[23] Gottfried JA, and Dolan RJ. The nose smells what the eye sees: crossmodal visual facilitation of human olfactory perception. Neuron 39: 375-386, 2003

[24] Morrot G, Brochet F, and Dubourdieu D. The color of odors. Brain Lang 79: 309-320, 2001.

[25] Österbauer RA, Matthews PM, Jenkinson M, Beckmann CF, Hansen PC, and Calvert GA. Color of scents: chromatic stimuli modulate odor responses in the human brain. J Neurophysiol 93: 3434-3441, 2005.

[26] Zellner DA, Bartoli AM, and Eckard R. Influence of color on odor identification and liking ratings. Am J Psychol 104: 547-561, 1991.

[27] Anderson AK, Christoff K, Stappen I, Panitz D, Ghahremani DG, Glover G, Gabrieli JD, and Sobel N. Dissociated neural representations of intensity and valence in human olfaction. Nat Neurosci 6: 196-202, 2003.

[28] de Araujo IE, Rolls ET, Kringelbach ML, McGlone F, and Phillips N. Taste-olfactory convergence, and the representation of the pleasantness of flavour, in the human brain. Eur J Neurosci 18: 2059-2068, 2003.

[29] de Araujo IE, Rolls ET, Velazco MI, Margot C, and Cayeux I. Cognitive modulation of olfactory processing. Neuron 46: 671-679, 2005.

[30] Gottfried JA, O'Doherty J, and Dolan RJ. Encoding predictive reward value in human amygdala and orbitofrontal cortex. Science 301: 1104-1107, 2003.

[31] Ishai A. Sex, beauty and the orbitofrontal cortex. Int J Psychophysiol 63: 181-185, 2006

[32] Schneider W, Eschman A, and Zuccolotto A. E-Prime reference guide. Pittsburgh, PA: Psychology Software Tools Inc. 2002.

[33] Jenkinson M, Bannister P, Brady M, and Smith S. Improved optimization for the robust and accurate linear registration and motion correction of brain images. Neuroimage 17: 825-841, 2002.

[34] Woolrich MW, Ripley BD, Brady M, and Smith SM. Temporal autocorrelation in univariate linear modeling of FMRI data. Neuroimage 14: 1370-1386, 2001.

[35] Beckmann CF, Jenkinson M, and Smith SM. General multilevel linear modeling for group analysis in FMRI. Neuroimage 20: 1052-1063, 2003.

[36] Forman SD, Cohen JD, Fitzgerald M, Eddy WF, Mintun MA, and Noll DC. Improved assessment of significant activation in functional magnetic resonance imaging (fMRI): use of a cluster-size threshold. Magn Reson Med 33: 636-647, 1995. 
[37] Friston KJ, Worsley KJ, Frakowiak RSJ, Mazziotta JC, and Evans AC. Assessing the significance of focal activations using their spatial extent. Hum Brain Mapp 1: 214-220, 1994.

[38] Worsley KJ, Evans AC, Marrett S, and Neelin P. A three-dimensional statistical analysis for CBF activation studies in human brain. J Cereb Blood Flow Metab 12: 900-918, 1992.

[39] O'Doherty J, Rolls ET, Francis S, Bowtell R, and McGlone F. Representation of pleasant and aversive taste in the human brain. J Neurophysiol 85: 1315-1321, 2001.

[40] Rolls ET, Kringelbach ML, and de Araujo IE. Different representations of pleasant and unpleasant odours in the human brain. Eur J Neurosci 18: 695-703, 2003.

[41] Zald DH, Lee JT, Fluegel KW, and Pardo JV. Aversive gustatory stimulation activates limbic circuits in humans. Brain 121: 1143-1154, 1998.

[42] Zald DH, and Pardo JV. Emotion, olfaction, and the human amygdala: amygdala activation during aversive olfactory stimulation. Proc Natl Acad Sci USA 94: 4119-4124, 1997.

[43] Royet JP, Hudry J, Zald DH, Godinot D, Gregoire MC, Lavenne F, Costes N, and Holley A. Functional neuroanatomy of different olfactory judgments. Neuroimage 13: 506-519, 2001.

[44] Ursu, S. and Carter, C. S. Outcome representations, counterfactual comparisons and the human orbitofrontal cortex: implications for neuroimaging studies of decisionmaking. Cogn Brain Res, 23(1), 51-60, 2005

[45] Liu X, Powell DK, Wang H, Gold BT, Corbly CR, and Joseph JE. Functional dissociation in frontal and striatal areas for processing of positive and negative reward information. J Neurosci 27:4587-4597, 2007

[46] Elloit R, Agnew Z, and Deakin B. Hedonic and Informational Functions of the Human Orbitofrontal Cortex. Cerebral Cortex 20(1): 198-204, 2010

[47] Winston JS, Gottfried JA, Kilner JM, and Dolan RJ. Integrated neural representations of odor intensity and affective valence in human amygdala. J Neurosci 25: 8903-8907, 2005.

[48] Dolan RJ, Morris JS, and de Gelder B. Crossmodal binding of fear in voice and face. Proc Natl Acad Sci USA 98: 10006-10010, 2001.

[49] Kesler-West ML, Andersen AH, Smith CD, Avison MJ, Davis CE, Kryscio RJ, and Blonder LX. Neural substrates of facial emotion processing using fMRI. Brain Res Cogn Brain Res 11: 213-226, 2001.

[50] Nitschke JB, Sarinopoulos I, Mackiewicz KL, Schaefer HS, and Davidson RJ. Functional neuroanatomy of aversion and its anticipation. Neuroimage 29: 106-116, 2006. 
[51] Sato W, Yoshikawa S, Kochiyama T, and Matsumura M. The amygdala processes the emotional significance of facial expressions: an fMRI investigation using the interaction between expression and face direction. Neuroimage 22: 1006-1013, 2004.

[52] Wicker B, Keysers C, Plailly J, Royet JP, Gallese V, and Rizzolatti G. Both of us disgusted in my insula: the common neural basis of seeing and feeling disgust. Neuron 40: 655-664, 2003. 
